\title{
Factors that predict a positive response on gonadotropin-releasing hormone stimulation test for diagnosing central precocious puberty in girls
}

\author{
Junghwan Suh, MD', \\ Myung Hyun Choi, MD', \\ Ah Reum Kwon, MD', \\ Ye Jin Kim, MD', \\ Jin Woo Jeong, MD', \\ Jung Min Ahn, MD', \\ Hyun Wook Chae, MD', \\ Duk Hee Kim, MD, PhD², \\ Ho-Seong Kim, MD, PhD ${ }^{1}$ \\ ${ }^{1}$ Department of Pediatrics, \\ Endocrine Research Institute, \\ Yonsei University College of \\ Medicine, Seoul, \\ ${ }^{2}$ Sowha Children's Hospital, Seoul, \\ Korea
}

\begin{abstract}
Purpose: The rapid increase in the incidence of precocious puberty in Korea has clinical and social significance. Gonadotropin-releasing hormone $(\mathrm{GnRH})$ stimulation test is required to diagnose central precocious puberty (CPP), however this test is expensive and time-consuming. This study aimed to identify factors that can predict a positive response to the GnRH stimulation test.

Methods: Clinical and laboratory parameters, including basal serum luteinizing hormone (LH), follicle-stimulating hormone (FSH), and estradiol (E2), were measured in 540 girls with clinical signs of CPP.

Results: Two hundred twenty-nine of 540 girls with suspected CPP had a peak serum $\mathrm{LH}$ level higher than $5 \mathrm{IU} / \mathrm{L}$ (the CPP group). The CPP group had advanced bone age $(P<0.001)$, accelerated yearly growth rate $(P<0.001)$, increased basal levels of $\mathrm{LH}$ $(P=0.02)$, FSH $(P<0.001), \mathrm{E} 2(P=0.001)$, and insulin-like growth factor-l levels $(P<0.001)$ compared to the non-CPP group. In contrast, body weight $(P<0.001)$ and body mass index $(P<0.001)$ were lower in the CPP group. Although basal LH was significantly elevated in the CPP group compared to the non-CPP group, there was considerable overlap between the 2 groups. Cutoff values of basal LH (0.22 IU/L) detected CPP with $87.8 \%$ sensitivity and $20.9 \%$ specificity.

Conclusion: No single parameter can predict a positive response on the $\mathrm{GnRH}$ stimulation test with both high sensitivity and specificity. Therefore, multiple factors should be considered in evaluation of sexual precocity when deciding the timing of the $\mathrm{GnRH}$ stimulation test.
\end{abstract}

Keywords: Precocious puberty, Diagnosis, Gonadotropin-releasing hormone, Forecasting, Female

\section{Introduction}

Revised: 25 October, 2013

Accepted: 18 Noverber, 2013

Address for correspondence: Ho-Seong Kim, MD, PhD

Department of Pediatrics, Endocrine Research Institute, Severance Children's Hospital, Yonsei University College of Medicine, 50 Yonsei-ro, Seodaemun-gu, Seoul 120-752, Korea

Tel: +82-2-2228-2069

Fax: +82-2-393-9118

E-mail:kimho@yuhs.ac
Precocious puberty is defined as the development of secondary sexual characteristics before 8 years of age in girls and 9 years of age in boys. Most cases result from inappropriate early activation of the hypothalamic-pituitary-gonadal (HPG) axis, termed central precocious puberty $(\mathrm{CPP})^{1)}$. Most cases of CPP are idiopathic CPP, which is diagnosed after excluding identifiable causes such as brain lesions, tumors, hydrocephalus, or head trauma ${ }^{2}$. The incidence of sexual precocity is increasing rapidly in Korea, and has recently been recognized as an emerging issue ${ }^{3}$. CPP can compromise height potential or result in psychosocial disturbances for those affected ${ }^{4,5)}$. It has been reported that early-maturing girls display more problem behaviors because of the gap between their and their peers physical appearance ${ }^{5)}$. Therefore, careful evaluation of pubertal signs, followed by early diagnosis and proper treatment, are important for the girls with suspected sexual precocity ${ }^{6}$. The diagnosis of CPP can be made by documentation of early activation of HPG axis in a child with progressive 
sexual development, accelerated growth rate, and advanced bone maturation. Currently, the gonadotropin-releasing hormone (GnRH) stimulation test is the standard method used to verify the activation of HPG axis ${ }^{7}$. However, the GnRH stimulation test requires multiple blood sampling over long time periods, and sometimes, it needs to be repeated multiple times before a diagnosis of CPP can be rendered. Therefore, we investigated the clinical and laboratory factors that predict positive results on the GnRH stimulation test because knowledge of these factors may help determine the patient selection and timing for the test.

This study aimed to identify factors that can predict a positive response on GnRH stimulation test in girls with suspected precocious puberty. Clinical and laboratory characteristics of the patients with precocious puberty were analyzed at the time of GnRH stimulation test. In addition, the predictive values of basal LH, follicle-stimulating hormone (FSH), and estradiol (E2) levels for diagnosis of CPP were examined.

\section{Materials and methods}

This study enrolled 540 girls who visited the Pediatric Endocrinology Clinic at Severance Children's Hospital for suspected CPP from March 2007 to June 2011. All subjects had onset of breast development before 8 years of age, accelerated growth rate, and bone age (BA) advancement at least 1 year compared to chronological age (CA). GnRH stimulation test was performed before 9 years of age. Among all subjects, 124 cases performed second GnRH stimulation test at 6-month interval, and only the data from last test were included. All subjects were evaluated for the following clinical and biochemical characteristics, such as pubertal stage, BA, CA, height, height standard deviation score (SDS), weight, weight SDS, body mass index (BMI, $\mathrm{kg} / \mathrm{m}^{2}$ ), BMI SDS, yearly growth rate, basal LH, basal FSH, basal E2, insulin-like growth factor-I (IGF-I), IGF-I SDS, IGF binding protein-3 (IGFBP-3), and IGFBP-3 SDS. In 124 subjects followed up for 6 months for second GnRH stimulation test, ratio of increase of BA to increase of CA (delta BA/delta CA) was investigated. Breast development was determined according to the Marshall and Tanner staging system $^{8)}$. BA was determined on the basis of the Greulich and Pyle method ${ }^{9)}$, by performing radiography of the left hand and left wrist at the GnRH stimulation test. The height, weight, BMI, height SDS, weight SDS, and BMI SDS were calculated using the Korean children and adolescents' growth standard ${ }^{10)}$. IGF-I SDS and IGFBP-3 SDS were converted from reference values to account for $\mathrm{CA}^{11)}$. During the GnRH stimulation test, the level of LH and FSH were checked just before GnRH administration, and then again at 30,60, 90, and 120 minutes after injection of $100 \mathrm{~g}$ of GnRH. Serum levels of basal LH and FSH were determined using the sequential 2-step immunoenzymatic assay (Access hLH, FSH Reagent Pack, Beckman Coulter Inc., Brea, CA, USA) with an intra-assay coefficient of variation (CV) of $3.5-5.4 \%$, inter-assay CV of $4.3-6.4 \%$, and a lower limit of detection of $0.2 \mathrm{IU} / \mathrm{L}$ for both gonadotropins. Serum E2 levels were measured using the radioimmunoassay (Coat-A-Count
Estradiol, Siemens, Erlangen, Germany) with an intra-assay CV of $4.0-7.0 \%$, inter-assay CV of $4.2-8.1 \%$, and a lower limit of detection of $8 \mathrm{pg} / \mathrm{mL}$. Serum IGF-I and IGFBP-3 levels were measured by chemiluminescence immunoassay (Liaison IGF-I Reagent, Liaison, Atlanta, GA, USA). CPP was diagnosed when the peak LH level was $\geq 5 \mathrm{IU} / \mathrm{L}$ on GnRH stimulation test. Other endocrine disorders in subjects or the presence of intracranial lesions detected by sella magnetic resonance imaging (MRI) were excluded. This study was approved by the Institutional Review Board of Severance Hospital (No. 4-2013-0377).

Statistical analyses were performed using IBM SPSS ver 20.0 (IBM Co., Armonk, NY, USA). Student $t$-test was used to compare the parameters between the groups with positive and negative results on the GnRH stimulation test. The receiver operating characteristic (ROC) curve analysis was used to assess the sensitivity and specificity of the variable factors. $P<0.05$ were considered statistically significant.

\section{Results}

\section{Comparison of clinical and biochemical characteristics between girls with and without CPP}

Of the 540 girls enrolled in this study, 229 had a positive result on the GnRH stimulation test and were therefore diagnosed with CPP, whereas the remaining 311 girls had a negative result. Height SDS was similar in both the CPP and non-CPP groups $(0.92 \pm 0.89$ vs. $0.84 \pm 0.95, P=0.34)$. Weight SDS $(0.53 \pm 0.77$ vs. $0.79 \pm 0.89, P<0.001)$ and BMI SDS $(0.09 \pm 0.92$ vs. $0.54 \pm 1.04$, $P<0.001)$ were lower in the CPP group than in the non-CPP group. Yearly growth rate was higher in the CPP group than in the non-CPP group $(8.88 \pm 2.91 \mathrm{~cm} / \mathrm{yr}$ vs. $6.36 \pm 2.20 \mathrm{~cm} / \mathrm{yr}, P$ $<0.001)$. BA advancement was higher in the CPP group than in the non-CPP group $(10.11 \pm 0.64 \mathrm{yr}$ vs. $9.74 \pm 0.97 \mathrm{yr}, P<0.001)$, but the difference between the BA and the CA (BA-CA) was not significant between the 2 groups $(1.81 \pm 0.63$ years vs. $1.78 \pm 0.72$ years, $P=0.59$ ) (Table 1 ). Ratio of increase of BA to increase of CA (delta BA/delta CA) was not different between the 2 groups (1.16 \pm 0.94 vs. $0.99 \pm 0.85, P=0.43$ ) (Table 1 ).

Basal LH levels $(0.93 \pm 1.07 \mathrm{IU} / \mathrm{L}$ vs. $0.75 \pm 0.61 \mathrm{IU} / \mathrm{L}, P=0.02)$ and basal FSH levels $(3.53 \pm 1.84 \mathrm{IU} / \mathrm{L}$ vs. $2.06 \pm 1.06 \mathrm{IU} / \mathrm{L}, P$ $<0.001)$ were higher in the CPP group than in the non-CPP group (Table 1). However, there was a considerable overlap between the 2 groups. Notably, only 45 of the 311 non-CPP girls (14.5\%) had basal LH levels less than $0.2 \mathrm{IU} / \mathrm{L}$ (the minimum detectable level) (Fig. 1). In contrast, 20 of the 229 girls with CPP (8.7\%) had basal LH levels less than 0.2 IU/L (Fig. 1). The basal LH/FSH ratio was lower in the CPP group than in the non-CPP group $(0.36 \pm 0.63$ vs. $0.47 \pm 0.58, P=0.03)$. Basal E2 level $(10.59 \pm 4.44 \mathrm{pg} / \mathrm{mL}$ vs. $9.09 \pm 2.93 \mathrm{pg} / \mathrm{mL}, P=0.001)$ and IGF-I SDS $(0.30 \pm 0.94$ vs. $-0.06 \pm 0.80, P<0.001)$ were higher in the CPP group than in the non-CPP group, whereas IGFBP-3 SDS was similar between the 2 groups $(-0.50 \pm 0.98$ vs. $-0.51 \pm 0.89$, $P=0.88$ ) (Table 1) 


\section{Regression analysis of clinical and biochemical parameters}

The clinical and biochemical parameters considered to be

Table 1. Clinical and biochemical parameters in patients with precocious puberty

\begin{tabular}{|c|c|c|c|}
\hline Characteristic & $\begin{array}{c}\text { CPP } \\
(n=229)\end{array}$ & $\begin{array}{l}\text { Non-CPP } \\
(n=311)\end{array}$ & $P$-value \\
\hline Height (cm) & $132.4 \pm 6.4$ & $129.7 \pm 7.0$ & $<0.001$ \\
\hline Height SDS & $0.92 \pm 0.89$ & $0.84 \pm 0.95$ & 0.340 \\
\hline Yearly growth rate (cm/yr) & $8.88 \pm 2.91$ & $6.36 \pm 2.20$ & $<0.001$ \\
\hline Bone age (yr) & $10.11 \pm 0.64$ & $9.74 \pm 0.97$ & $<0.001$ \\
\hline Chronological age (yr) & $8.30 \pm 0.69$ & $7.96 \pm 0.91$ & $<0.001$ \\
\hline$B A-C A(y r)$ & $1.81 \pm 0.63$ & $1.78 \pm 0.72$ & 0.590 \\
\hline$\triangle B A / \triangle C A^{a)}$ & $1.16 \pm 0.94$ & $0.99 \pm 0.85$ & 0.430 \\
\hline Weight (kg) & $29.87 \pm 5.02$ & $30.19 \pm 5.84$ & 0.510 \\
\hline Weight SDS & $0.53 \pm 0.77$ & $0.79 \pm 0.89$ & $<0.001$ \\
\hline Body mass index $\left(\mathrm{kg} / \mathrm{m}^{2}\right)$ & $16.99 \pm 2.05$ & $17.89 \pm 2.54$ & $<0.001$ \\
\hline Body mass index SDS & $0.09 \pm 0.92$ & $0.54 \pm 1.04$ & $<0.001$ \\
\hline Tanner stage & $2.50 \pm 0.58$ & $2.29 \pm 0.56$ & $<0.001$ \\
\hline Basal LH (IU/L) & $0.93 \pm 1.07$ & $0.75 \pm 0.61$ & 0.020 \\
\hline Basal FSH (IU/L) & $3.53 \pm 1.84$ & $2.06 \pm 1.06$ & $<0.001$ \\
\hline Basal LH/FSH ratio & $0.36 \pm 0.63$ & $0.47 \pm 0.58$ & 0.030 \\
\hline Basal estradiol (pg/mL) & $10.59 \pm 4.44$ & $9.09 \pm 2.93$ & 0.001 \\
\hline |GF-I (ng/mL) & $270.5 \pm 85.6$ & $231.5 \pm 71.4$ & $<0.001$ \\
\hline IGF-ISDS & $0.30 \pm 0.94$ & $-0.06 \pm 0.80$ & $<0.001$ \\
\hline IGFBP-3 ( $\mu \mathrm{g} / \mathrm{mL})$ & $2.65 \pm 0.53$ & $2.60 \pm 0.48$ & 0.300 \\
\hline IGFBP-3 SDS & $-0.50 \pm 0.98$ & $-0.51 \pm 0.89$ & 0.880 \\
\hline Peak LH (IU/L) & $15.73 \pm 13.66$ & $3.25 \pm 1.01$ & $<0.001$ \\
\hline Peak FSH (IU/L) & $18.47 \pm 7.66$ & $15.98 \pm 5.99$ & $<0.001$ \\
\hline
\end{tabular}

Values are presented as mean \pm standard deviation.

CPP, central precocious puberty; SDS, standard deviation score; LH, luteinizing hormone; FSH, follicle-stimulating hormone; IGF-I, insulinlike growth factor-I; IGFBP-3, insulin-like growth factor binding protein-3.

${ }^{\text {a) }} \mathrm{CPP}(\mathrm{n}=48)$, non-CPP $(\mathrm{n}=76)$.

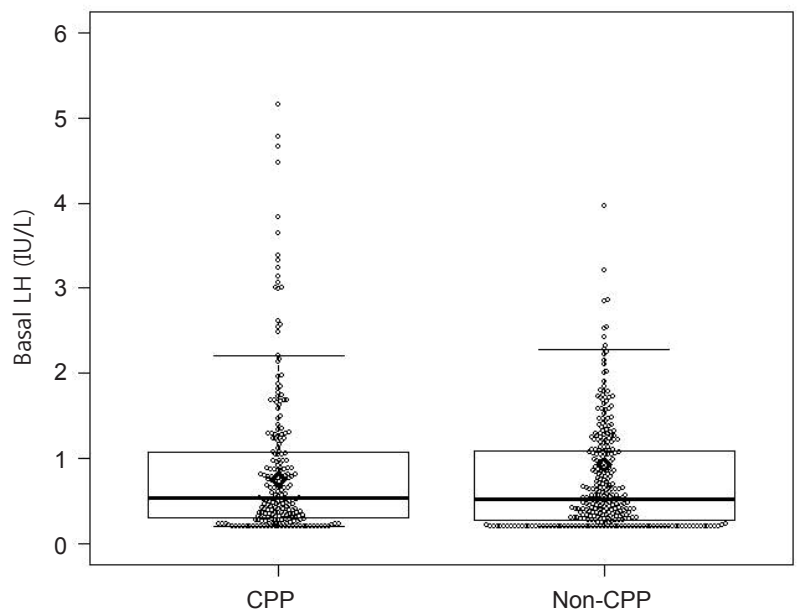

Fig. 1. Results show considerable overlap between the 2 groups. CPP, central precocious puberty; LH, luteinizing hormone. related to the results of GnRH stimulation testing (Table 1) were adjusted using binary logistic regression analysis. After regression analysis, basal FSH (odds ratio [OR], 2.18; 95\% confidence interval $[\mathrm{CI}], 1.749-2.708 ; P<0.001)$, yearly growth rate (OR, 1.49; 95\% CI: $1.3431-1.659 ; P<0.001)$, IGF-I SDS (OR, 1.32; 95\% CI, 1.002-1.737; $P=0.048$ ), and BMI SDS (OR, $0.58 ; 95 \% \mathrm{CI}, 0.372-0.899 ; P=0.015)$ were significantly related to a positive response on $\mathrm{GnRH}$ stimulation, whereas basal $\mathrm{LH}$, basal E2, and weight SDS were not (Table 2).

\section{Determination of cutoff values for basal LH, FSH, and E2 from ROC curves}

The optimal cutoff values for basal LH, FSH, and E2 to discriminate between girls with and without CPP were determined using ROC curves. The optimal cutoff value of basal LH was $0.22 \mathrm{IU} / \mathrm{L}$, with a sensitivity of $87.8 \%$ and a specificity

Table 2. Binary logistic regression analysis of the parameters related to $\mathrm{GnRH}$ stimulation test results

\begin{tabular}{lccc}
\hline Variable & Odds ratio & $95 \% \mathrm{Cl}$ & $P$-value \\
\hline Weight SDS & 0.97 & $0.571-1.629$ & 0.893 \\
Body mass index SDS & 0.58 & $0.372-0.899$ & 0.015 \\
Yearly growth rate & 1.49 & $1.341-1.659$ & $<0.001$ \\
Basal LH & 1.09 & $0.833-1.415$ & 0.542 \\
Basal FSH & 2.18 & $1.749-2.708$ & $<0.001$ \\
Basal estradiol & 1.05 & $0.951-1.150$ & 0.353 \\
IGF-I SDS & 1.32 & $1.002-1.737$ & 0.048 \\
\hline
\end{tabular}

$\mathrm{Cl}$, confidence interval; SDS, standard deviation score; LH, luteinizing hormone; FSH, follicle-stimulating hormone; IGF-I, insulin-like growth factor-l.

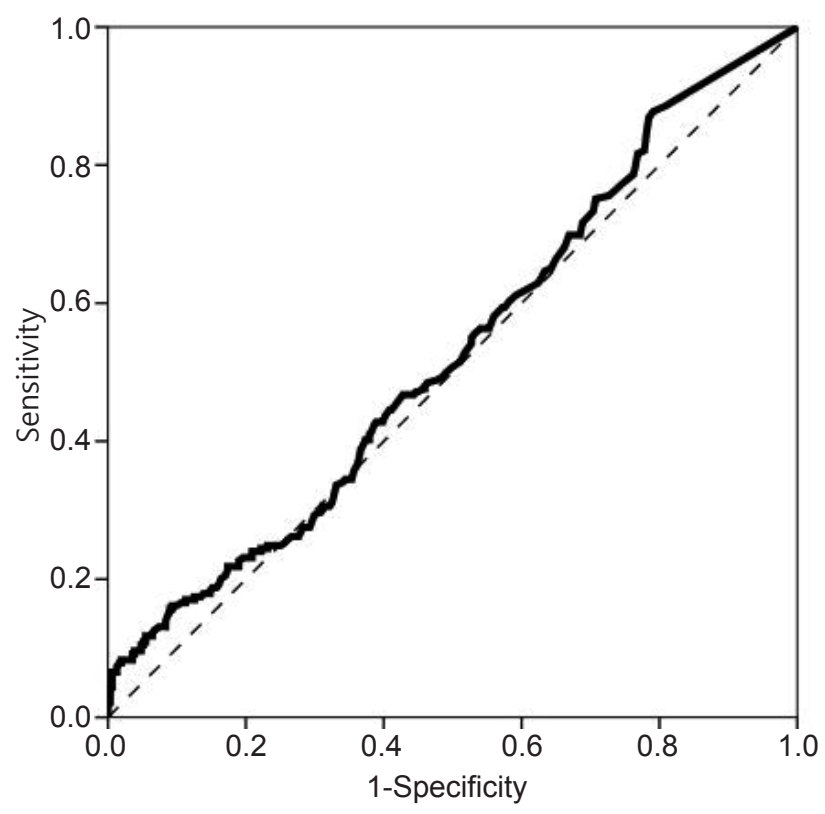

Fig. 2. The statistically optimal cutoff value of basal luteinizing hormone was 0.22 IU/L, with a sensitivity of $87.8 \%$ and a specificity of $20.9 \%$ (area under the receiver operating characteristic curve, 0.529 ). 
of $20.9 \%$ (area under the ROC curve, 0.529) (Fig. 2). The cutoff value of basal FSH was $2.30 \mathrm{IU} / \mathrm{L}$, with a sensitivity of $76.4 \%$ and a specificity of $65.9 \%$ (area under the ROC curve, 0.78) (Fig. 3). The cutoff value of basal E2 was $8.30 \mathrm{pg} / \mathrm{mL}$, with a sensitivity of $53.3 \%$ and a specificity of $65.6 \%$ (area under the ROC curve, 0.615) (Fig. 4).

\section{Discussion}

CPP is the most common form of precocious puberty and often results in adverse effects on both physical growth and psychological maturation. Therefore, an early diagnosis followed by immediate treatment is essential to improve both the physical and mental well-being of girls with CPP. Currently, GnRHstimulated LH levels are the gold standard diagnostic biomarker of CPP. However, GnRH stimulation testing is expensive, timeconsuming, and often requires repeated tests before a definitive diagnosis can be made. To avoid unnecessary testing and patient discomfort and to reduce costs, several previous studies have attempted to identify clinical and laboratory biomarkers that may be used to screen patients with suspected CPP in order to guide the proper timing of GnRH testing.

These studies reported that basal LH levels could alone diagnose CPP accurately. LH level could be a useful parameter because testing of LH level is simple, entails minimal patient discomfort, and is quicker to perform compared to the $\mathrm{GnRH}$ stimulation test. Neely et al. ${ }^{12)}$ have reported that in 49 girls with clinical signs suggestive of CPP, spontaneous LH levels correlated strongly with peak stimulated LH levels. Therefore, they suggested that LH level could be a useful screening tool for CPP. They demonstrated that spontaneous LH levels in excess of

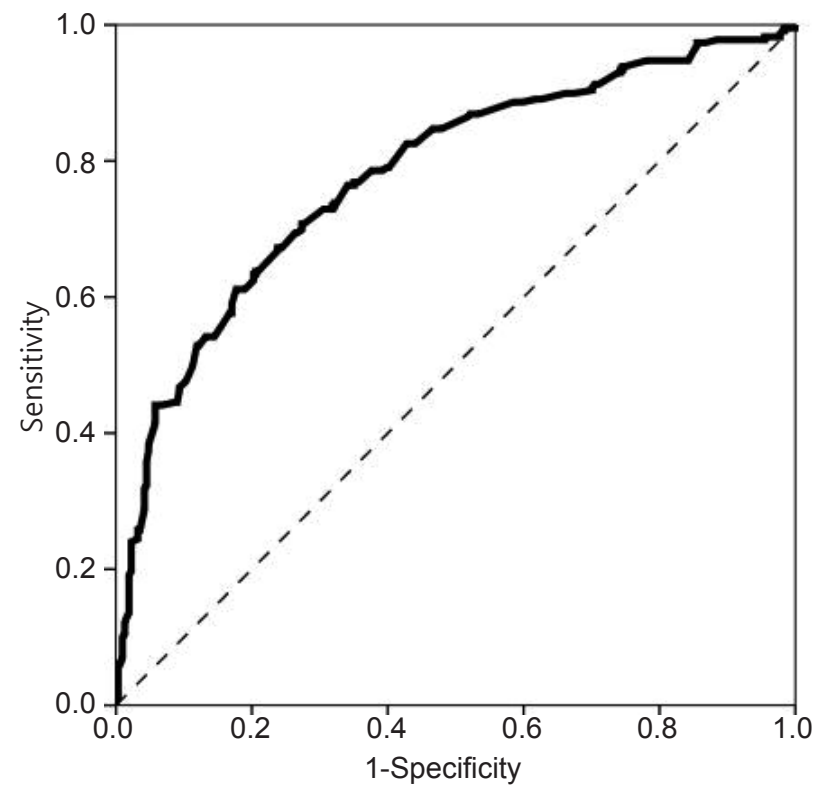

Fig. 3. The cutoff value of basal follicle-stimulating hormone was $2.30 \mathrm{IU} / \mathrm{L}$, with a sensitivity of $76.4 \%$ and a specificity of $65.9 \%$ (area under the receiver operating characteristic curve, 0.780 ).
$0.1 \mathrm{IU} / \mathrm{L}$ predicted CPP with $94 \%$ sensitivity and $88 \%$ specificity. Moreover, random LH levels in excess of 0.3 IU/L had 100\% specificity for CPP. Similarly, Houk et al. ${ }^{13)}$ investigated basal LH levels in 55 girls with suspected CPP using 2 chemiluminescent assays (Delfia, Wallac Oy, Turku, Finland; Architect, Abbott Park, IL, USA), and assessed the utility of using a single sample to diagnose CPP. They reported that a basal LH cutoff value of 0.83 $\mathrm{U} / \mathrm{L}$ in the Delfia assay had a sensitivity of $93 \%$ and a specificity of $100 \%$, whereas a cutoff value of $1.05 \mathrm{U} / \mathrm{L}$ in the Architect assay had a sensitivity of $100 \%$ and a specificity of $100 \%$. They therefore suggested that a single basal LH measurement is adequate to document a pubertal state in most-but not allgirls with CPP. Additionally, Pasternak et al. ${ }^{14)}$ reported that a basal LH cutoff value of $0.1 \mathrm{IU} / \mathrm{L}$ had a specificity of $94.7 \%$ and a sensitivity of $64.4 \%$, suggesting that a single basal LH measurement is adequate to confirm, but not to refute, the presence of CPP.

In our study, basal LH was significantly higher in the CPP group than in the non-CPP group. However, only $14.5 \%$ of non-CPP patients had basal LH levels less than $0.2 \mathrm{IU} / \mathrm{L}$ (the minimum detectable level), whereas $8.7 \%$ of CPP patients did, suggesting there was a considerable overlap between the 2 groups. Moreover, a basal LH cutoff value of $0.22 \mathrm{IU} / \mathrm{L}$ determined from ROC curves had a sensitivity of $87.8 \%$ and a specificity of $20.9 \%$, suggesting that basal LH levels do not have a sufficiently high sensitivity or specificity to accurately diagnose CPP. Our results also demonstrated that basal FSH, basal E2, and the basal LH/FSH ratio do not have predictive value for the diagnosis of CPP. A basal FSH cutoff value of $2.30 \mathrm{IU} / \mathrm{L}$ and a basal E2 cutoff value of $8.30 \mathrm{pg} / \mathrm{mL}$ had sensitivities of $76.4 \%$ and $53.3 \%$ and specificities of $65.9 \%$ and $65.6 \%$, respectively. The

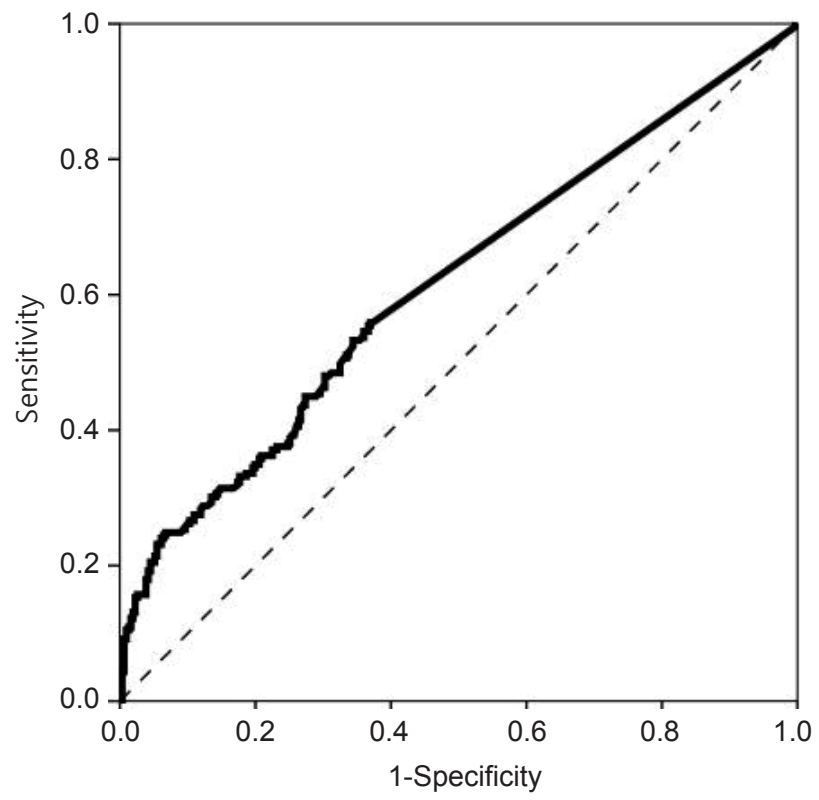

Fig. 4. The cutoff value of basal estradiol was $8.30 \mathrm{pg} / \mathrm{mL}$, with a sensitivity of $53.3 \%$ and a specificity of $65.6 \%$ (area under the receiver operating characteristic curve, 0.615). 
basal LH/FSH ratio is considered to be elevated in girls with $\mathrm{CPP}^{15)}$. In contrast, our results showed that the basal LH/FSH ratio was significantly higher in the non-CPP group than in the CPP group, and an appropriate cutoff value for basal LH/FSH ratio that had both high sensitivity and high specificity could not be determined. Thus, a single laboratory parameter that can predict a positive result on GnRH stimulation with both high sensitivity and high specificity could not be determined in our study. This contradiction to the findings of previous studies may relate to the difference in subject numbers between studies. A previous study using a larger cohort ${ }^{16)}$ also reported that a single basal LH measurement was not adequate to confirm CPP. Another large cohort study composed of 803 girls reported that a basal LH cutoff value of $1.1 \mathrm{IU} / \mathrm{L}$ had a sensitivity of $69.1 \%$ and a specificity of $50.5 \%{ }^{17}$. These results from large cohort studies, including ours, suggest that a single basal LH measurement is not specific or sensitive enough to diagnose CPP and should therefore not be used as a screening test alone.

Furthermore, we assessed many clinical and laboratory parameters that may predict a positive result by $\mathrm{GnRH}$ stimulation test in this study. We found that BA, yearly growth rate, serum IGF-I levels, and IGF-I SDS were higher in girls with CPP than in those without CPP, whereas weight SDS, BMI, and BMI SDS were lower in girls with CPP. In addition, binary logistic regression analysis showed that accelerated yearly growth rate, high IGF-I SDS, and low BMI SDS were significantly correlated with a positive result on the $\mathrm{GnRH}$ stimulation test. The most reliable clinical measurement was accelerated yearly growth rate, in accordance with the findings of a previous study in a large cohor ${ }^{16)}$. However, use of yearly growth rate has limitations because at least 3 months are required to assess the growth velocity. Furthermore, we could not determine a proper cutoff value to diagnose CPP using the yearly growth rate. BA advancement and ratio of increase of BA to increase of CA also could not be used to diagnose CPP in our study because these parameters were observed in both groups to the same degree. Several studies have reported that serum IGF-I levels were elevated in girls with CPP, suggesting that serum IGF-I levels are related to $\mathrm{CPP}^{16,18)}$. In our study, we analyzed IGF-I SDS to adjust the value according to sex and age using reference values from Korean children and adolescents ${ }^{11)}$. Binary logistic regression analysis showed that serum IGF-I SDS was significantly correlated with CPP, but its predictive value was weak. Previous studies have reported that obesity is associated with earlier pubertal maturation and therefore might be one of the reasons for the general trend towards earlier pubertal onset $^{19,20)}$. However, obesity is also reported to be associated with early pubertal maturation in girls but delayed pubertal onset in boys $^{21)}$, so the exact relationship between obesity and precocious puberty is not fully determined. In our study, low BMI SDS was significantly correlated with a positive result on the GnRH stimulation test.

In conclusion, the present study demonstrated that girls with CPP had an accelerated growth rate, advanced BA, and increased basal gonadotropin and IGF-1 levels. We were unable to identify one individual biochemical parameter that could accurately predict a positive result on the GnRH stimulation test. Therefore, we suggest that multiple factors, such as clinical findings and basal gonadotropin levels, should be considered when evaluating sexual precocity and determining the proper timing for GnRH stimulation testing.

\section{Conflict of interest}

No potential conflict of interest relevant to this article was reported.

\section{Acknowledgments}

This work was supported by Grant from Inje University, 2011.

\section{References}

1. Merke DP, Cutler GB Jr. Evaluation and management of precocious puberty. Arch Dis Child 1996;75:269-71.

2. Cisternino M, Arrigo T, Pasquino AM, Tinelli C, Antoniazzi F, Beduschi L, et al. Etiology and age incidence of precocious puberty in girls: a multicentric study. J Pediatr Endocrinol Metab 2000;13 Suppl 1:695-701.

3. Hur K, Park M. Five year national trend of precocious puberty in Korean children, 2004-2008. Program and Abstracts, the 58th Annual Fall Meeting of the Korean Pediatric Society; 2009 Oct 23-24; Seoul, Korea. Seoul: Korean Pediatric Society; 2009:179.

4. Brauner R, Adan L, Malandry F, Zantleifer D. Adult height in girls with idiopathic true precocious puberty. J Clin Endocrinol Metab 1994;79:415-20.

5. Tremblay L, Frigon JY. Precocious puberty in adolescent girls: a biomarker of later psychosocial adjustment problems. Child Psychiatry Hum Dev 2005l;36:73-94.

6. Berberoğlu M. Precocious puberty and normal variant puberty: definition, etiology, diagnosis and current management. J Clin Res Pediatr Endocrinol 2009;1:164-74.

7. Lee PA. Laboratory monitoring of children with precocious puberty. Arch Pediatr Adolesc Med 1994;148:369-76.

8. Marshall WA, Tanner JM. Variations in pattern of pubertal changes in girls. Arch Dis Child 1969;44:291-303.

9. Greulich WW, Pyle SI. Radiographic atlas of skeletal development of the hand and wrist. 2nd ed. Stranford (CA): Stranford University Press, 1959.

10. Korea Centers for Disease Control and Prevention, Division of Chronic Disease Surveillance, Committee for the Development of Growth Standard for Korean Children and Adolescents; Korean Pediatric Society, Committee for School Health and Public Health Statistics. 2007 Korean children and adolescents growth standard (commentary for the development of 2007 growth chart). Cheongwon: Korea Centers for Disease Control and Prevention, Division of Chronic Disease Surveillance, 2007. 
11. Hyun SE, Lee BC, Suh BK, Chung SC, Ko CW, Kim HS, et al. Reference values for serum levels of insulin-like growth factor-I and insulin-like growth factor binding protein-3 in Korean children and adolescents. Clin Biochem 2012;45:16-21.

12. Neely EK, Wilson DM, Lee PA, Stene M, Hintz RL. Spontaneous serum gonadotropin concentrations in the evaluation of precocious puberty. J Pediatr 1995;127:47-52.

13. Houk CP, Kunselman AR, Lee PA. Adequacy of a single unstimulated luteinizing hormone level to diagnose central precocious puberty in girls. Pediatrics 2009;123:e1059-63.

14. Pasternak Y, Friger M, Loewenthal N, Haim A, Hershkovitz E. The utility of basal serum LH in prediction of central precocious puberty in girls. Eur J Endocrinol 2012;166:2959.

15. Supornsilchai V, Hiranrat P, Wacharasindhu S, Srivuthana S, Aroonparkmongkol S. Basal luteinizing hormone/ follicle stimulating hormone ratio in diagnosis of central precocious puberty. J Med Assoc Thai 2003;86 Suppl 2:S145-51.
16. Nam HK, Rhie YJ, Son CS, Park SH, Lee KH. Factors to predict positive results of gonadotropin releasing hormone stimulation test in girls with suspected precocious puberty. J Korean Med Sci 2012;27:194-9.

17. Lee HS, Park HK, Ko JH, Kim YJ, Hwang JS. Utility of Basal luteinizing hormone levels for detecting central precocious puberty in girls. Horm Metab Res 2012;44:851-4.

18. Sørensen K, Aksglaede L, Petersen JH, Andersson AM, Juul A. Serum IGF1 and insulin levels in girls with normal and precocious puberty. Eur J Endocrinol 2012;166:903-10.

19. Biro FM, Lucky AW, Simbartl LA, Barton BA, Daniels SR, Striegel-Moore R, et al. Pubertal maturation in girls and the relationship to anthropometric changes: pathways through puberty. J Pediatr 2003;142:643-6.

20. Burt Solorzano CM, McCartney CR. Obesity and the pubertal transition in girls and boys. Reproduction 2010;140:399-410.

21. Wang Y. Is obesity associated with early sexual maturation? A comparison of the association in American boys versus girls. Pediatrics 2002;110:903-10. 